\title{
An ontology-based search engine for protein-protein interactions
} Byungkyu Park and Kyungsook Han*

\author{
Address: School of Computer Science and Engineering, Inha University, Incheon 402-751, South Korea \\ E-mail: Byungkyu Park - bpark@inhaian.net; Kyungsook Han* - khan@inha.ac.kr \\ *Corresponding author
}

from The Eighth Asia Pacific Bioinformatics Conference (APBC 2010)

Bangalore, India 18-2I January 2010

Published: 18 January 2010

BMC Bioinformatics 2010, I I(SuppI I):S23 doi: I0.II86/I47I-2I05-II-SI-S23

This article is available from: http://www.biomedcentral.com/I47I-2/05/II/SI/S23

(C) 2010 Park and Han; licensee BioMed Central Ltd.

This is an open access article distributed under the terms of the Creative Commons Attribution License (http://creativecommons.org/licenses/by/2.0), which permits unrestricted use, distribution, and reproduction in any medium, provided the original work is properly cited.

\begin{abstract}
Background: Keyword matching or ID matching is the most common searching method in a large database of protein-protein interactions. They are purely syntactic methods, and retrieve the records in the database that contain a keyword or ID specified in a query. Such syntactic search methods often retrieve too few search results or no results despite many potential matches present in the database.

Results: We have developed a new method for representing protein-protein interactions and the Gene Ontology (GO) using modified Gödel numbers. This representation is hidden from users but enables a search engine using the representation to efficiently search protein-protein interactions in a biologically meaningful way. Given a query protein with optional search conditions expressed in one or more GO terms, the search engine finds all the interaction partners of the query protein by unique prime factorization of the modified Gödel numbers representing the query protein and the search conditions.

Conclusion: Representing the biological relations of proteins and their $\mathrm{GO}$ annotations by modified Gödel numbers makes a search engine efficiently find all protein-protein interactions by prime factorization of the numbers. Keyword matching or ID matching search methods often miss the interactions involving a protein that has no explicit annotations matching the search condition, but our search engine retrieves such interactions as well if they satisfy the search condition with a more specific term in the ontology.
\end{abstract}

\section{Background}

Recent advances in high-throughput interaction detection methods such as yeast two-hybrid and mass spectrometry techniques have led to a rapid expansion of protein-protein interaction data in several organisms. For example, there are about 8, 000 interactions between
4, 000 S. cerevisiae proteins, approximately 24, 000 interactions between 7,600 D. melanogaster proteins, and over 5, 100 interactions between 2, 900 C. elegans proteins [1]. More than 137, 000 protein interactions and 60, 000 genetic interactions are also known for six major model organisms [2]. Several databases have been 
built for the large amount of protein-protein interaction data, which include BOND [3], DIP [4], MIPS [5], HPRD [6], HPID [7] and BioGRID [2]. Most of the databases allow the user to retrieve protein-protein interactions that satisfy a condition specified in a query. Keyword matching or ID matching is one of the most commonly used searching methods. This type of search retrieves all of the records in the database which contain a keyword or ID specified in a query. The user can alter retrieval results using Boolean operators such as AND, OR and NOT.

However, a search method based on keyword matching or ID-matching is purely syntactic and does not consider biological relations between the keywords or IDs. If the user gets too many protein-protein interactions, selecting the protein-protein interactions to focus on entirely relies on the discretion of the user. If the user gets too few protein-protein interactions or no results at all, the user will probably have to look for other resources. For example, BOND returns 5, 100 protein-protein interactions for a keyword query of 'ATP binding' whereas it returns only 96 interactions for a keyword query of 'nucleotide binding'. The term 'nucleotide binding' is at a higher level than 'ATP binding' in the Gene Ontology (GO) hierarchy [8], but it returns much fewer search results than 'ATP binding'. This search anomaly occurs because the search method of BOND does not consider the biological relation between keywords. Besides, the user must enter an exact keyword or ID in the query since BOND and many other protein-protein interaction databases do not support the 'autocomplete' feature when searching the databases.

Recently a few ontology-based information retrieval methods have developed for biological literature or databases [9-11], but little work has been reported on the ontology-based search for protein-protein interactions in databases. We developed a new representation of the Gene Ontology (GO) and a search engine that finds all the semantically relevant interactions of a query protein using the representation. For a GO term, all the GO terms at the lower level than the GO term in the GO hierarchy are automatically considered when searching for protein-protein interactions. For example, when dealing with a query like "for protein $\mathrm{p}$ annotated with a GO term $\mathrm{f}$, find the interaction partners of $\mathrm{p}$ ", the search engine considers not only the GO annotation $f$ but also all the GO annotations below $\mathrm{f}$ in the GO hierarchy. Several computational methods have been developed to elucidate protein function from the analysis of protein-protein interaction data [12], and our search engine will be useful to identify proteins with common function or subcellular localization. This paper presents the development of a new representation method of protein-protein interactions and a search engine for protein-protein interactions using the representation method.

\section{Methods}

Gödel numbers are typically used to uniquely encode any list of positive integers $\left\{a_{1}, a_{2}, \ldots, a_{n}\right\}$ by

$$
\varphi_{a_{1} a_{2} \ldots a_{n}}=\prod_{k=1}^{n} p_{k}^{a_{k}},
$$

where $p_{k}$ is the $k$ th prime number [13].

However, the original Gödel numbers defined by equation (1) cannot represent the Directed Acyclic Graph (DAG) structure of the Gene Ontology. Consider an example shown in Table 1, in which a unique natural number represents a term and a Gödel number represents the relation between the terms. Term 4 is a kind of Term 2 by Relation R3, and Term4 is a kind of Term 1 by Relation R1. But these relations cannot be inferred unambiguously from the representation of Table 1.

Therefore, we modify the Gödel numbers as follows:

1. Assign each term a prime number instead of a natural number using Algorithms 1 and 2 .

2. Represent each relation between the terms by a modified Gödel number using Algorithm 3. The modified Gödel number is the product of the prime numbers representing the terms in the relation and their ancestors, including the root term in the Gene Ontology hierarchy.

For example, relation R3 of Table 2 is represented by 42, which has prime factors of 2 (Term1, root node in the

Table I: Gödel number representation. Original Gödel numbers cannot represent the Directed Acyclic Graph (DAG) structure of the Gene Ontology

\begin{tabular}{lcll}
\hline Term & $\begin{array}{c}\text { Natural } \\
\text { number }\end{array}$ & Relation & Gödel Number \\
\hline is a & 1 & & \\
part of & 2 & RI: Term2 is a Term I & $2^{4} 3^{1} 5^{3}=6,000$ \\
Term I & 3 & R2: Term3 is part of Term2 & $2^{5} 3^{2} 5^{4}=180,000$ \\
Term2 & 4 & R3: Term4 is a Term2 & $2^{6} 3^{1} 5^{4}=120,000$ \\
Term3 & 5 & & \\
Term4 & 6 & & \\
\hline
\end{tabular}

The following example shows why the original Gödel numbers fail to represent the GO structure. Suppose that we represent terms by unique natural numbers and the relations between them by Gödel numbers. In this example, Term 4 is a kind of Term 2 by relation R3, and Term 4 is a kind of Term I by relation RI. But these relations cannot be inferred from the representation because the original Gödel numbers are not sufficient to represent the DAG structure of GO. 
Table 2: Modified Gödel number representation

\begin{tabular}{lcll}
\hline Term & $\begin{array}{c}\text { Prime } \\
\text { number }\end{array}$ & Relation & Modified Gödel Number \\
\hline Term I & 2 & RI: Term2 & relation(is a) $=3 \times 2=6$ \\
Term2 & 3 & $\begin{array}{l}\text { is a Term I } \\
\text { R2: Term3 } \\
\text { is part of } \\
\text { Term2 } \\
\text { R3: Term4 }\end{array}$ & relation(part of) $=5 \times 3 \times 2=30$ \\
is a Term2 & 5 & relation(is a) $=7 \times 3 \times 2=42$ \\
Term4 & 7 & & \\
\hline
\end{tabular}

Each term is assigned a prime number instead of natural number, and each relation is denoted by a modified Gödel number, which is a multiplication of prime numbers representing the term and its ancestors in the ontology hierarchy. For example, relation R3 is denoted by 42 , which has prime factors 2 (Term I, root node in the hierarchy), 3

(Term2), and 7 (Term3). Using this representation, relation R3 can be easily inferred by unique factorization of it into primes (Term1, Term2, and Term3).

hierarchy), 3 (Term2), and 7 (Term3). This representation enables us to infer the meaning of $\mathrm{R} 3$ by unique factorization of R3 into prime numbers representing Term1, Term2, and Term3.

Table 3 shows an example of predicting protein-protein interactions from domain-domain interactions using the representation. Suppose that domainA interacts with domainB (R4 in Table 3), proteinA has domainA (R5), and that protein $B$ has domainB (R6). These relations can be represented by modified Gödel numbers, as shown in Table 3. Using this representation, the hypothesis that proteinA interacts with proteinB can be tested by simple arithmetic operations such as integer division and modulo operation.

$$
\begin{gathered}
143=11(\text { domain } A) \times 13(\text { domain } B) \\
46,189=187(\text { Protein } A) \times 247(\text { Protein } B) \\
\\
46,189 \equiv 0(\bmod 143)
\end{gathered}
$$

In this example, the relation that domainA interacts with domainB is represented by a modified Gödel number 143, which is the product of 11 (representing domain A) and 13 (domainB) (equation 2). The hypothesis that proteinA interacts with protein $B$ is represented by a modified Gödel number 46, 189 from the multiplication of 187 (proteinA) by 247 (proteinB) (equation 3). Since the remainder after dividing 46, 189 (representing the hypothesis that proteinA interacts with proteinB) by 143 (representing the relation that domainA interacts with domainB) is 0 (equation 4), the hypothesis that proteinA interacts with proteinB turns out to be true.

\section{Algorithm 1 Generate modified Gödelnumbers}

This algorithm assigns prime numbers to GO terms, stores the relation of the GO terms in a local DB by calling Algorithm 2, and generates the modified Gödel numbers by multiplying the prime numbers.

$1: T=\left\{t_{1}, t_{2}, \ldots, t_{G}\right\}\{G$ is the number of GO terms. $\}$

2: $P=\left\{p_{1}, p_{2}, \ldots, p_{G}\right\}\{P$ is an ordered set of prime numbers. $\}$

3: for all $i \in\{1,2, \ldots, G\}$ do

4: $\quad t_{i} \leftarrow p_{i}$ \{Assign a prime number to a GO term. $\}$

5: end for

6: for all $t \in\left\{t_{1}, t_{2}, \ldots, t_{G}\right\}$ do

7: Algorithm2(t. key, $t)$ \{Store the prime number assignment in a local DB.\}

8: end for

9: for all $t \in\left\{t_{1}, t_{2}, \ldots, t_{G}\right\}$ do

10: Modified Gödel number $\leftarrow$ Algorithm3(t. key)

11: end for

Table 3: Reasoning protein-protein interactions

\begin{tabular}{lcll}
\hline Term & $\begin{array}{c}\text { Prime } \\
\text { number }\end{array}$ & Relation & Modified Gödel Number \\
\hline domainA & 11 & & \\
domainB & 13 & R4: domainA interacts with domainB & $\begin{array}{l}\text { relation(interacts) }=11 \times 13=143 \\
\text { ProteinA }\end{array}$ \\
ProteinB & 17 & R5: ProteinA has domainA & relationas $=17 \times 11=187$ \\
& 19 & R6: ProteinB has domainB & relation $($ has $=19 \times 13=247$ \\
\hline
\end{tabular}

Using our representation, it is possible to infer protein-protein interactions from domain-domain interactions. Suppose that domainA interacts with domainB, proteinA has domainA, and that proteinB has domainB. Simple arithmetic operations such as integer division and modulo operations are sufficient to infer that ProteinA interacts with ProteinB. 


\section{Algorithm 2 StoreRepresentation(Term.key, Term)}

This algorithm stores the prime number assignment of the GO term and its parents by a recursive call until the parameter Term is a root term of the hierarchy.

1: if Term.isRoot $==$ false then

2: for all $t \in$ Term.parent do

3: $\quad$ SetRelation(Term.key, t.key, t.prime) \{Store the prime numbers assigned to Term and its parents.\}

4: Algorithm2(Term.key, $t$ ) \{Recursive call for the parent of Term $\}$

5: end for

6: end if

Algorithm 3 ModifiedGödelNumber(Term.key)

This algorithm calculates a modified Gödel number by multiplying the prime numbers representing the parameter Term.key and its ancestor terms in the ontology hierarchy.

1: ArrayList list $=$ GetRelation $($ Term.key $)$ \{ Retrieve the prime numbers for Term and its ancestors. $\}$

2: $v a r=1$

3: for all $t \in$ list do

4: $\quad$ var $=$ var $\times$ t.prime

5: end for

6: return var

\section{Results and discussion}

\section{User interface of the search engine}

A prototype of the ontology-based search engine has been implemented in the $\mathrm{C \#}$ programming language [21]. We generated more than 26, 000 prime numbers using the Sieve of Eratosthenes [14], and used the Java BigInteger class to store the numbers and to perform multiplication and modulo operations on them. When the user specifies a GO term or protein superfamily [15] for the query protein, the search engine returns all interactions that involve the protein annotated with the GO term or superfamily as well as the proteins annotated with more specific terms than the specified GO term. To make the search engine easy to use, it provides autocomplete functionality for $\mathrm{GO}$ terms or protein superfamilies. So, a partial term entered by the user is expanded into one or more complete GO terms or superfamilies that are consistent with the partial term. An example of using the autocomplete functionality for GO terms in the search engine is shown in Figure 1.

The user can also get protein-protein interactions from the web service of the search engine using the Web Services Description Language (WSDL). WSDL is an XML format for describing network services as a set of endpoints operating on messages containing either document-oriented or procedure-oriented information [16]. The interaction data returned by the search engine can be displayed and saved in the PSI-MI format [17] or in the PSI-MI format with XML style sheets.

\section{Comparison of the search methods}

We tested the ontology-based search engine on the interaction data of HPRD [6] and compared it with the ID-matching search method. Table 4 shows the number of protein-protein interactions found in HPRD by the two search methods. HPRD release 7 contains 38, 190 interactions between 8,800 human proteins, which are annotated with 470 GO terms. The total number of GO terms is more than 26,000 , but only $1.8 \%$ of the 26,000 GO terms are used for annotating human proteins in HPRD. Our search engine can infer up to 698 GO terms for human proteins from the $470 \mathrm{GO}$ terms that were used for annotating human proteins (see the supplementary material at [21]).

The GO term ID of GO:0008150 is the root node of the GO hierarchy for biological process. With a query of GO:0008150, the ontology-based search engine found 36,523 interactions $(95.6 \%$ of the total 38,190 interactions of HPRD), but the ID-matching search retrieved only 5 interactions $(0.01 \%$ of the total 38 , 190 interactions of HPRD). With a query of GO:0008152 for metabolic process, which is the descendent node of GO:0008150 in the GO hierarchy (Figure 2), the ontology-based search engine found 19, 828 interactions $(51.9 \%$ of the total 38,190 interactions of HPRD), but the ID-matching search found 2, 862 interactions (7.5\% of the total 38, 190 interactions). The ID-matching search returned more search results with a more specific term than with a less specific one. The IDmatching search found no interactions with a query of GO:0044238 or GO:0043170, but found 5, 324 interactions with a query of GO:0019538, which is at a lower level than GO:0044238 or GO:0043170. These search anomalies occur because the ID-matching search method does a purely syntactic search and does not consider the 
An Ontology-Based Search Engine for Protein-Protein Interactions

News $\mid$ Search | OnlinePrediction | RDF | WSDL | Supplementary materials | Help

HPID: Search

\begin{tabular}{|c|c|c|}
\hline Gene Ontolos & GO:000584 & Search \\
\hline We have developed a new me & $\mathrm{GO}, 0005826$ & \\
\hline odfied Godel numbers. This representation is hid & GO:0005824 & $\begin{array}{l}\text { as and the Gene Ontology (GO) ustng } \\
\text {; a search engine using the representation to }\end{array}$ \\
\hline iciently search protein-protein interactions in a bi & GO:0005828 & Given a query protein with optional search \\
\hline conditions expressed in one or more GO terms, the & $\begin{array}{l}G O: 0005821 \\
G O \cdot 0005823\end{array}$ & nds all the interaction partners of the query \\
\hline protein by prime factorization of the modified $\mathrm{G} \delta$ & $\mathrm{GO}: 0005827$ & query protein and the search conditions. \\
\hline hile keyword matching $\circ \mathrm{D}$ matching search : & GO:0005825 & involving a protein with no explcit \\
\hline & GO:0005829 & eractions as well if they satisfy the \\
\hline
\end{tabular}

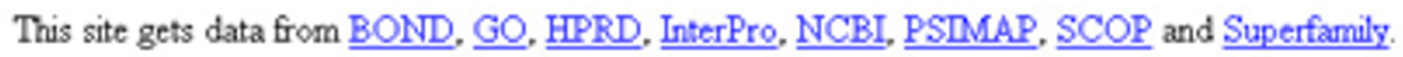

Department of Computer Science and Information Engineering. Inha University, Incheon 402-751, Korea (c) (1) $(9)$ 82-32-860-7388 (phone), 82-32-863-4386 (tax), Kyangsook Han (email)

This work is licensed under a Creative Commons Attribution-Noncommercial-No Derivative Works 2.0 Korea License.

Figure I

User interface of the ontology-based search engine. An example of using the autocomplete functionality for GO terms.

Table 4: Comparison of search results by two search methods

\begin{tabular}{|c|c|c|c|}
\hline GO term ID & GO term name & ID-matching search & Ontology-based search \\
\hline \multicolumn{4}{|c|}{ Biological process } \\
\hline GO:0008I50 & biological process & $5(0.01 \%)$ & $36,523(95.63 \%)$ \\
\hline GO:0008I52 & metabolic process & 2, $862(7.49 \%)$ & $19,828(51.92 \%)$ \\
\hline GO:0044238 & primary metabolic process & $0(0.00 \%)$ & $17,434(45.65 \%)$ \\
\hline GO:0043170 & macromolecule metabolic process & $0(0.00 \%)$ & $7,2 \mid I(\mid 8.88 \%)$ \\
\hline GO:0019538 & protein metabolic process & $5,324(13.94 \%)$ & $5,659(14.82 \%)$ \\
\hline \multicolumn{4}{|c|}{ Molecular function } \\
\hline GO:0003676 & nucleic acid binding & $10(0.03 \%)$ & $8,733(22.87 \%)$ \\
\hline GO:0003677 & DNA binding & I, 944 (5.09\%) & $6,935(18.16 \%)$ \\
\hline GO:0003700 & transcription factor activity & $5,164(13.52 \%)$ & $5,164(13.52 \%)$ \\
\hline \multicolumn{4}{|c|}{ Cellular component } \\
\hline GO:0005622 & intracellular & $0(0.00 \%)$ & 31,694 (82.99\%) \\
\hline GO:0005737 & cytoplasm & $17,312(45.33 \%)$ & 20,990 (54.96\%) \\
\hline GO:0005829 & cytosol & $452(1.18 \%)$ & $452(1.18 \%)$ \\
\hline
\end{tabular}

The number of protein-protein interactions found in HPRD release 7 by each search method. The numbers inside parentheses indicate the ratio of the interactions to the total 38, 190 interactions of HPRD. The ID-matching search often finds more interactions with a specialized GO terms than with a less specialized terms since it does not consider semantic relation between ontology terms.

relation of GO terms at all. In contrast, the ontologybased search finds interactions not only by the GO term specified in the query but by specialized terms of the term.
Figure 3 shows the interaction networks of human proteins, which were found by the two search methods and visualized by Cytoscape [18]. Networks 1-11 of Figure 3 represent the 70 protein-protein interactions 


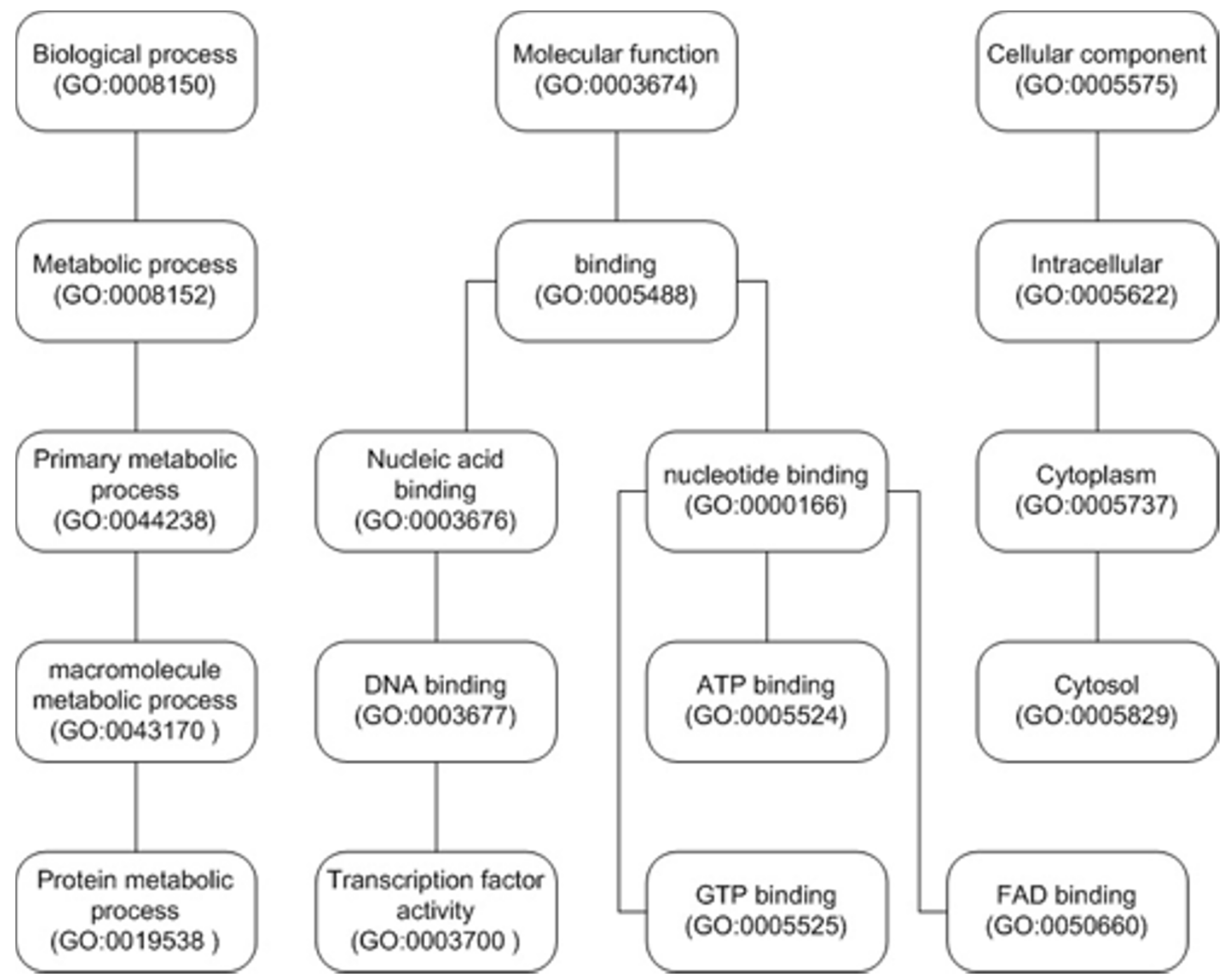

Figure 2

Example of the gene ontology hierarchy. A partial view of the three ontologies of the Gene Ontology (GO). The GO terms 'biological process', 'molecular function', and 'cellular component' are the root nodes of three GO hierarchies. Several intermediate terms between the nodes are not shown for clarity.

found by the ontology-based search with the query of 'Nucleotide binding' (the GO term ID GO:0000166) from the HPRD data. As shown in Table 5, only 5 out of the 70 interactions involve a protein with an explicit annotation of 'Nucleotide binding'. The remaining 65 interactions were inferred from the Gene Ontology by finding a protein annotated with a more specialized term such as 'ATP binding', 'FAD binding' or 'GTP binding' than 'Nucleotide binding' (see Figure 2 for the partial view of the Gene Ontology of molecular function).

Networks $7-11$ of Figure 3 represent the 31 interactions found by the ontology-based search with the query of 'ATP binding' (GO:0005524). 'ATP binding' is at the lower level than 'Nucleotide binding' in the ontology hierarchy, and therefore it is quite reasonable that the search results with 'ATP binding' are exclusively included in the search results with 'Nucleotide binding'. On the contrary, the ID-matching search found only 5 interactions (networks 5-6) with the query of 'Nucleotide binding' and missed the remaining 65 interactions. But with the query of 'ATP binding' the ID-matching search found the same 31 interactions (networks 7-11) as those found by the ontology-based search.

The search engine also allows the user to specify multiple conditions on the query protein. Table 6 shows the search results by the two search methods when the user 
Table 5: Ontology-based search with 'Nucleotide binding' GO term

\begin{tabular}{|c|c|c|c|}
\hline $\begin{array}{l}\text { Query protein } \\
\text { HPRD_ID }\end{array}$ & Function & $\begin{array}{l}\text { Partner protein } \\
\text { HPRD_ID }\end{array}$ & Function \\
\hline HPRD_02944 & ATP binding & HPRD_0243I & Acyltransferase activity \\
\hline HPRD_0I368 & ATP binding & HPRD_02147 & ATP binding \\
\hline HPRD_0I368 & ATP binding & HPRD_02300 & ATPase activity \\
\hline HPRD_02|47 & ATP binding & HPRD_12171 & Catalytic activity \\
\hline HPRD_0I368 & ATP binding & HPRD_02110 & Extracellular matrix structural constituent \\
\hline HPRD_02944 & ATP binding & HPRD_02682 & GTPase activity \\
\hline HPRD_09468 & ATP binding & HPRD_08986 & Phospholipase activity \\
\hline HPRD_0I368 & ATP binding & HPRD_02610 & Protein binding \\
\hline HPRD_02944 & ATP binding & HPRD_03913 & Protein binding \\
\hline HPRD_09468 & ATP binding & HPRD_0I496 & Protein serine/threonine kinase activity \\
\hline HPRD_09468 & ATP binding & HPRD_02619 & Protein serine/threonine kinase activity \\
\hline HPRD_09468 & ATP binding & HPRD_03479 & Protein serine/threonine kinase activity \\
\hline HPRD_05802 & ATP binding & HPRD_04066 & Protein serine/threonine kinase activity \\
\hline HPRD_09468 & ATP binding & HPRD_05428 & Protein serine/threonine kinase activity \\
\hline HPRD_05802 & ATP binding & HPRD_02963 & Receptor activity \\
\hline HPRD_09468 & ATP binding & HPRD_0II58 & Structural constituent of myelin sheath \\
\hline HPRD_02|47 & ATP binding & HPRD_0I235 & Transcription regulator activity \\
\hline HPRD_09468 & ATP binding & HPRD_0059I & Translation regulator activity \\
\hline HPRD_09468 & ATP binding & HPRD_06774 & Translation regulator activity \\
\hline HPRD_09468 & ATP binding & HPRD_06802 & Translation regulator activity \\
\hline HPRD_09468 & ATP binding & HPRD_09084 & Translation regulator activity \\
\hline HPRD_0I368 & ATP binding & HPRD_0305I & Transporter activity \\
\hline HPRD_16742 & FAD binding & HPRD_II762 & DNA binding \\
\hline HPRD_16742 & FAD binding & HPRD_02171 & Hydrolase activity \\
\hline HPRD_04I00 & GTP binding & HPRD_07I35 & Acyltransferase activity \\
\hline HPRD_04I00 & GTP binding & HPRD_0I72I & Auxiliary transport protein activity \\
\hline HPRD_04100 & GTP binding & HPRD_0I722 & Auxiliary transport protein activity \\
\hline HPRD_04I00 & GTP binding & HPRD_04738 & GTP binding \\
\hline HPRD_04738 & GTP binding & HPRD_067I6 & GTP binding \\
\hline HPRD_II978 & GTP binding & HPRD_00743 & GTPase activity \\
\hline HPRD_II978 & GTP binding & HPRD_00766 & GTPase activity \\
\hline HPRD_04I00 & GTP binding & HPRD_03297 & GTPase activity \\
\hline HPRD_10360 & GTP binding & HPRD_03297 & GTPase activity \\
\hline HPRD_04I00 & GTP binding & HPRD_I2228 & GTPase activity \\
\hline HPRD_04738 & GTP binding & HPRD_I2228 & GTPase activity \\
\hline HPRD_10360 & GTP binding & HPRD_06692 & GTPase activity \\
\hline HPRD_I0360 & GTP binding & HPRD_08555 & GTPase activity \\
\hline HPRD_II978 & GTP binding & HPRD_09191 & GTPase activity \\
\hline HPRD_II978 & GTP binding & HPRD_09973 & GTPase activity \\
\hline HPRD_II978 & GTP binding & HPRD_II 820 & GTPase activity \\
\hline HPRD_10360 & GTP binding & HPRD_04398 & Protein binding \\
\hline HPRD_II 978 & GTP binding & HPRD_0I265 & Protein serine/threonine kinase activity \\
\hline HPRD_064I9 & GTP binding & HPRD_03384 & Protein serine/threonine phosphatase activity \\
\hline HPRD_04I00 & GTP binding & HPRD_06288 & RNA binding \\
\hline HPRD_04738 & GTP binding & HPRD_01853 & Structural constituent of cytoskeleton \\
\hline HPRD_04I00 & GTP binding & HPRD_0I45I & Structural molecule activity \\
\hline HPRD_064I9 & GTP binding & HPRD_01859 & Transcription factor activity \\
\hline HPRD_064I9 & GTP binding & HPRD_I65I5 & Transcription regulator activity \\
\hline HPRD_04100 & GTP binding & HPRD_03967 & Ubiquitin-specific protease activity \\
\hline HPRD_04738 & GTP binding & HPRD_03967 & Ubiquitin-specific protease activity \\
\hline HPRD_09704 & Nucleotide binding & HPRD_03356 & Signal transducer activity \\
\hline HPRD_09704 & Nucleotide binding & HPRD_I6544 & Transcription factor activity \\
\hline HPRD_09704 & Nucleotide binding & HPRD_0322I & Transcription regulator activity \\
\hline HPRD_I3II5 & Nucleotide binding & HPRD_030I5 & Unknown \\
\hline HPRD_09704 & Nucleotide binding & HPRD_04484 & Unknown \\
\hline
\end{tabular}

In HPRD the ontology-based search found 70 interactions involving a protein annotated with 'Nucleotide binding'. Only 5 interactions have a protein with an explicit annotation of 'Nucleotide binding'. The remaining 65 interactions were inferred by finding a protein annotated with a more specialized term such as 'ATP binding', 'FAD binding' or 'GTP binding'. Due to space limit, 15 interactions (7 self-interactions and 8 interactions involving a protein with unknown function) are not shown here. 


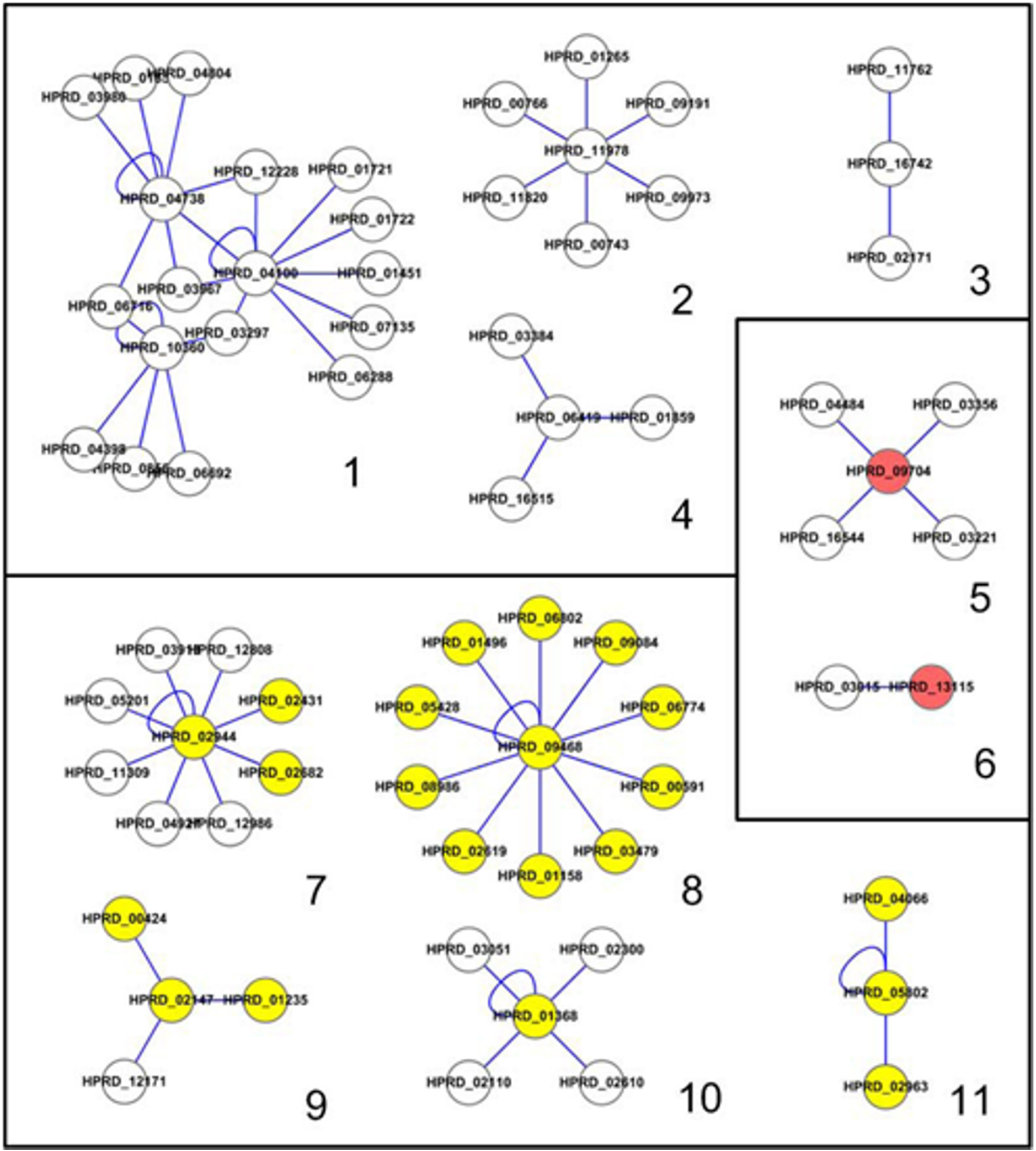

Figure 3

Interaction network of human proteins found with Nucleotide binding and ATP binding. Networks I - I represent the 70 protein-protein interactions found by the ontology-based search with the query of 'Nucleotide binding' from the HPRD data. Networks 7-II represent the 3I interactions found by the ontology-based search with the query of 'ATP binding', which is a more specific term than 'Nucleotide binding'. The ID-matching search found only 5 interactions (networks 5-6) with 'Nucleotide binding' and missed all the other interactions whereas its search results with 'ATP binding' are same as those of the ontology-based search (networks 7-II). Yellow nodes represent the human proteins explicitly annotated with 'ATP binding', pink nodes represent the human protein explicitly annotated with 'Nucleotide binding', and white nodes represent the human proteins with no explicit annotation of 'ATP binding' nor 'Nucleotide binding'. The GO term IDs of the proteins found by the search methods are listed in Table 5 . 
Table 6: Example of searching protein-protein interactions by specifying multiple $\mathrm{GO}$ terms on the query protein

\begin{tabular}{llrr}
\hline $\begin{array}{l}\text { Multiple GO terms } \\
\text { Query protein }\end{array}$ & $\begin{array}{r}\text { Search method } \\
\text { ID-matching } \\
\text { search }\end{array}$ & $\begin{array}{r}\text { Ontology- } \\
\text { based search }\end{array}$ \\
\hline $\begin{array}{l}\text { Biological } \\
\text { process }\end{array}$ & Cellular component & & \\
GO:0019538 & GO:0005737 & $1994(5.22 \%)$ & $3062(8.02 \%)$ \\
GO:0019538 & GO:0005576 & $753(1.97 \%)$ & $769(2.01 \%)$ \\
$\begin{array}{l}\text { Molecular } \\
\text { function }\end{array}$ & Cellular component & & \\
GO:0003700 & GO:0005737 & $576(1.51 \%)$ & $592(1.55 \%)$ \\
GO:0003700 & GO:0005576 & $6(0.02 \%)$ & $103(0.27 \%)$ \\
\hline
\end{tabular}

Search results when two GO terms are specified on the query protein, one for the biological process and another for the cellular component of the query protein. specifies two GO terms, 'protein metabolic process' (GO:0019538) and 'cytoplasm' (GO:0005737), as conditions on the query protein. The ontology-based search found more interactions than the ID-matching search for all queries in the table.

The implementation of the ontology-based search engine is not complete yet and being expanded to support various query types. For example, it will allow the user to search interactions by specifying GO terms both on the query and interaction partner proteins or by specifying multiple GO terms on the interaction partner protein. Figure 4 shows an interaction network between hepatitis $\mathrm{C}$ virus (HCV) and human proteins, which was constructed with the interaction data from a literature

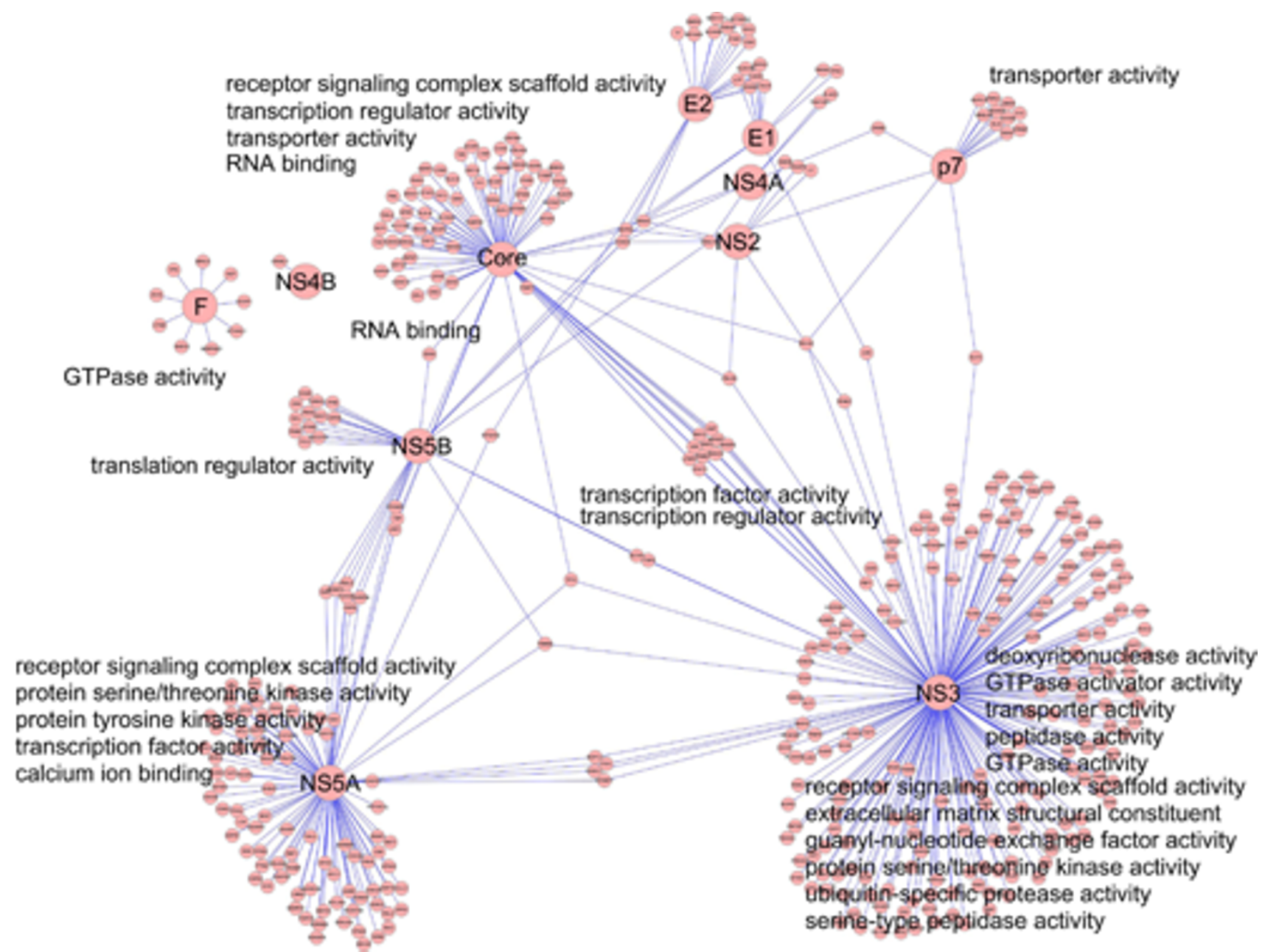

Figure 4

Interaction network of HCV-human proteins. The network contains HCV proteins (core, EI, E2, NS2, NS3, NS4A, NS4B, NS4A, NS5B, F and p7) and the human proteins interacting with them. The interaction data was obtained from a literature [19] and the network was visualized by Cytoscape [18]. The GO annotations for the HCV proteins and human proteins in the network are available at [2I]. 
[19]. Since the current search engine has the modified Gödel number representation for human proteins only, it cannot find interaction partners in other species yet. However, the search engine will be expanded to retrieve interactions between human proteins and other types of proteins. The Gene Ontology annotations for the HCV proteins and human proteins in the network of Figure 4 are available at [21].

\section{Conclusion}

We have developed a new method for representing protein-protein interactions and the Gene Ontology (GO) using modified Gödel numbers. This representation is hidden from users but enables a search engine to efficiently find protein-protein interactions in a biologically meaningful way. A prototype of the search engine is available at [21]. The search engine can find all interactions involving the query protein in almost realtime since the interaction partners of the query protein can be found unambiguously by prime factorization of the modified Gödel numbers representing the query protein and the search conditions.

The OWL Web Ontology Language [20] was established, but there have been no databases of protein-protein interactions that can process queries like "find every protein $\mathrm{p}$ with function $\mathrm{f}^{\text {" }}$ or "for every protein $\mathrm{p}$ with function $\mathrm{f}$, find the interaction partners of $\mathrm{p}$ ". To the best of our knowledge, the ontology-based search engine presented in this paper is the first one that can deal with such queries. This paper presented preliminary results of the ontology-based search engine, and it is being expanded into a full-featured search engine.

\section{Competing interests}

The authors declare that they have no competing interests.

\section{Authors' contributions}

Byungkyu Park conceived the idea of using modified Gödel numbers, implemented the system and prepared the first draft of the manuscript. Kyungsook Han supervised the work and rewrote the manuscript.

\section{Acknowledgements}

This work was supported by the National Research Foundation of Korea (2009-006647I).

This article has been published as part of BMC Bioinformatics Volume II Supplement 1, 2010: Selected articles from the Eighth Asia-Pacific Bioinformatics Conference (APBC 2010). The full contents of the supplement are available online at http://www.biomedcentral.com/I47I$\underline{2105 / 11 \text { ? issue }=S 1 \text {. }}$.

\section{References}

I. Chen J, Hsu W, Lee ML and Ng SK: Increasing confidence of protein interactomes using network topological metrics. Bioinformatics 2006, 22(16): 1998-2004.

2. Breitkreutz BJ, Stark C, Reguly T, Boucher L, Breitkreutz A, Livstone M, Oughtred R, Lackner DH, Bahler J and Wood V, et al: The BioGRID interaction database: 2008 update. Nucleic Acids Research 2008, 36:D637-D640.

3. Bader GD, Betel D and Hogue CWV: BIND: the Biomolecular Interaction Network Database. Nucleic Acids Research 2003 $31: 248-250$.

4. Xenarios I, Salwinski L, Duan XQJ, Higney P, Kim SM and Eisenberg D: DIP, the Database of Interacting Proteins: a research tool for studying cellular networks of protein interactions. Nucleic Acids Research 2002, 30:303-305.

5. Mewes HW, Dietmann S, Frishman D, Gregory R, Mannhaupt G, Mayer KFX, Munsterkotter M, Ruepp A, Spannagl M and Stuemp en V, et al: MIPS: analysis and annotation of genome information in 2007. Nucleic Acids Research 2008, 36:DI96-D20I.

6. Prasad TSK, Goel R, Kandasamy K, Keerthikumar S, Kumar S, Mathivanan S, Telikicherla D, Raju R, Shafreen B and Venugopal A, et al: Human Protein Reference Database-2009 update. Nucleic Acids Research 2009, 37:D767-D772.

7. Han K, Park B, Kim H, Hong J and Park J: HPID: The Human Protein Interaction Database. Bioinformatics 2004, 20:2466-2470.

8. Harris MA, Deegan JI, Lomax J, Ashburner M, Tweedie S, Carbon S, Lewis S, Mungall C, Day-Richter J and Eilbeck K, et al: The Gene Ontology project in 2008. Nucleic Acids Research 2008, 36: D440-D444.

9. Corsi C, Ferragina P and Marangoni R: The BioPrompt-box: an ontology-based clustering tool for searching in biological databases. BMC Bioinformatics 2007, 8(SuppI I).

10. Milward D, Bjareland M, Hayes W, Maxwell M, Oberg L, Tilford N, Thomas J, Hale R, Knight $S$ and Barnes JE: Ontology-based interactive information extraction from scientific abstracts. Comparative and Functional Genomics 2005, 6:67-71.

II. Muller HM, Kenny EE and Sternberg PW: Textpresso: An ontology-based information retrieval and extraction system for biological literature. Plos Biology 2004, 2: 1984-1998.

12. Sharan R, Ulitsky I and Shamir R: Network-based prediction of protein function. Molecular Systems Biology 2007, 3(13).

13. Gödel Number. http://mathworld.wolfram.com/GoedelNumber. html.

14. Sieve of Eratosthenes. http://mathworld.wolfram.com/SieveofEratosthenes.html.

15. Wilson D, Pethica R, Zhou YD, Talbot C Vogel C Madera M Chothia $C$ and Gough J: SUPERFAMILY-sophisticated comparative genomics, data mining, visualization and phylogeny. Nucleic Acids Research 2009, 37:D380-D386.

16. Web Services Description Language. http://www.w3.org/TR/ wsdl.

17. Hermjakob H, Montecchi-Palazzi L, Bader G, Wojcik R, Salwinski L, Ceol A, Moore S, Orchard S, Sarkans U and von Mering C, et al: The HUPOPSI's Molecular Interaction format - a community standard for the representation of protein interaction data. Nature Biotechnology 2004, 22(2): 177-183.

18. Shannon P, Markiel A, Ozier O, Baliga NS, Wang JT, Ramage D, Amin N, Schwikowski B and Ideker T: Cytoscape: A software environment for integrated models of biomolecular interaction networks. Genome Research 2003, 13:2498-2504.

19. de Chassey B, Navratil V, Tafforeau L, Hiet MS, Aublin-Gex A, Agaugue S, Meiffren G, Pradezynski F, Faria BF and Chantier T, et al: Hepatitis C virus infection protein network. Molecular Systems Biology 2008, 4(230).

20. OWL Web Ontology Language. http://www.w3.org/TR/owlfeatures.

21. A prototype system of the ontology-based search engine for protein-protein interactions. http://ontology.hpid.org. 